6 Colby TV, Koss MN, Travis WD. Miscellaneous mesenchymal tumors. In: Sobin LH, Rosai J, eds. Atlas of Tumor Pathology: Tumors of the Lower Respiratory Tract. Washington, Armed Forces Institute of Pathology, 1995; pp. 353-356.

7 Jauthzke G, Muller-Ruchholtz E, Thalmann U. Immunohistological detection of estrogen and progesterone receptors in multiple and well differentiated leiomyomatous lung tumors in women with uterine leiomyomas (so-called benign metastasizing leiomyomas). Pathol Res Pract 1996; 192: 215-223.

8 Arai T, Yasuda Y, Takaya T, et al. Natural decrease of benign metastasizing leiomyoma. Chest 2000; 117: 921.

9 Rivera JA, Christopoulos S, Small D, et al. Hormonal manipulation of benign metastasizing leiomyomas: report of two cases and review of the literature. J Clin Endocrinol Metab 2004; 89: 3183-3188.

10 Tietze L, Gunther K, Horbe A, et al. Benign metastasizing leiomyoma: a cytogenetically balanced but clonal disease. Hum Pathol 2000; 31: 126-128.

11 Patton KT, Cheng L, Papavero V, et al. Benign metastasizing leiomyoma: clonality, telomere length and clinicopathologic analysis. Mod Pathol 2006; 19: 130-140.

12 Nucci MR, Drapkin R, Dal Cin P, et al. Distinctive cytogenetic profile in benign metastasizing leiomyoma: pathogenetic implications. Am J Surg Pathol 2007; 31: 737-743.

\title{
Successful stenting of anastomotic stenosis of the left pulmonary artery after single lung transplantation
}

\section{To the Editor:}

Lung transplantation is an established therapy for a variety of end-stage lung diseases. Successful transplantation improves prognosis and quality of life in most recipients. In the current setting where lung donors are scarce, single-lung transplantation allows for more extensive utilisation of the limited donor organ pool [1]. Although forced expiratory volume in $1 \mathrm{~s}$ (FEV1) recovery is lower and the risk of bronchiolitis obliterans syndrome is higher, single lung transplant recipients still have comparable exercise tolerance and quality-of-life scores when compared to bilateral lung transplant recipients [2].

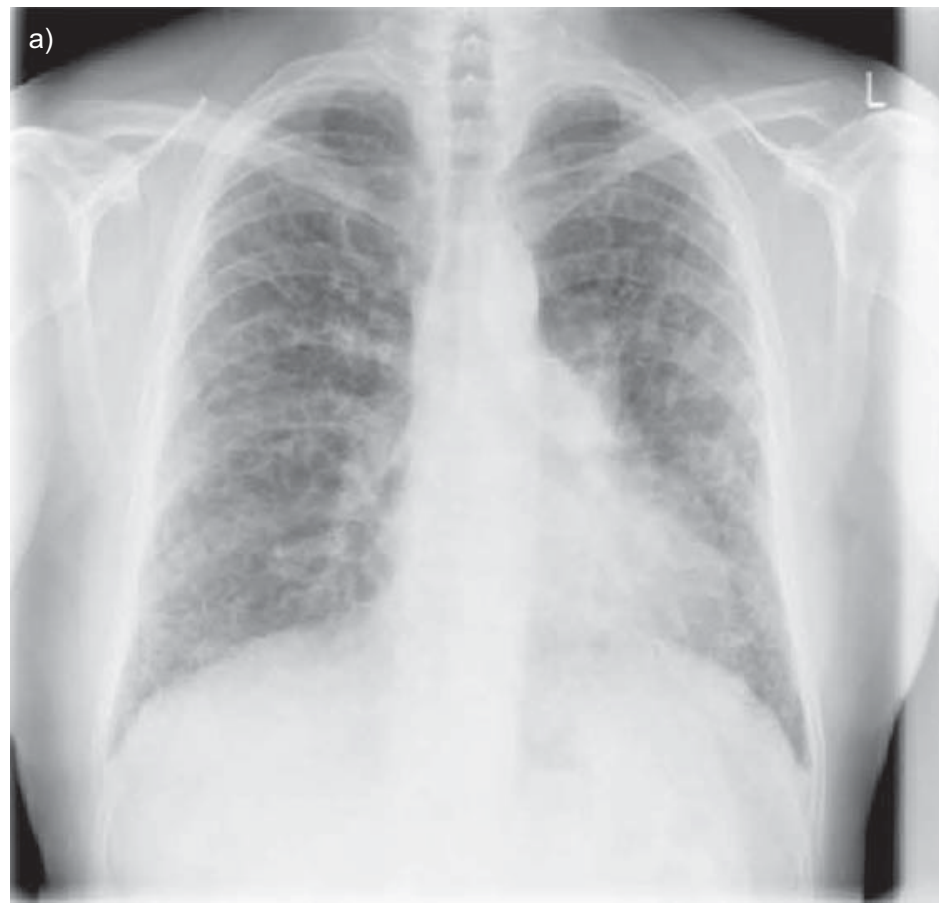

Fortunately, vascular anastomotic stenoses are an uncommon event following lung transplantation. There are two types of vascular complications: either pulmonary arterial stenosis or pulmonary venous stenosis. The structures affected determine the clinical manifestations: arterial obstruction leads to pulmonary ischaemia and infarction, and venous obstruction leads to pulmonary oedema. The diagnosis should be considered in the presence of unexplained exertional hypoxaemia and persistent pulmonary hypertension. The diagnosis can be confirmed with computed tomography pulmonary angiogram (CTPA) and pulmonary angiography. As the complication is rare there are no clear treatment algorithms. In the very early phase (1 week),

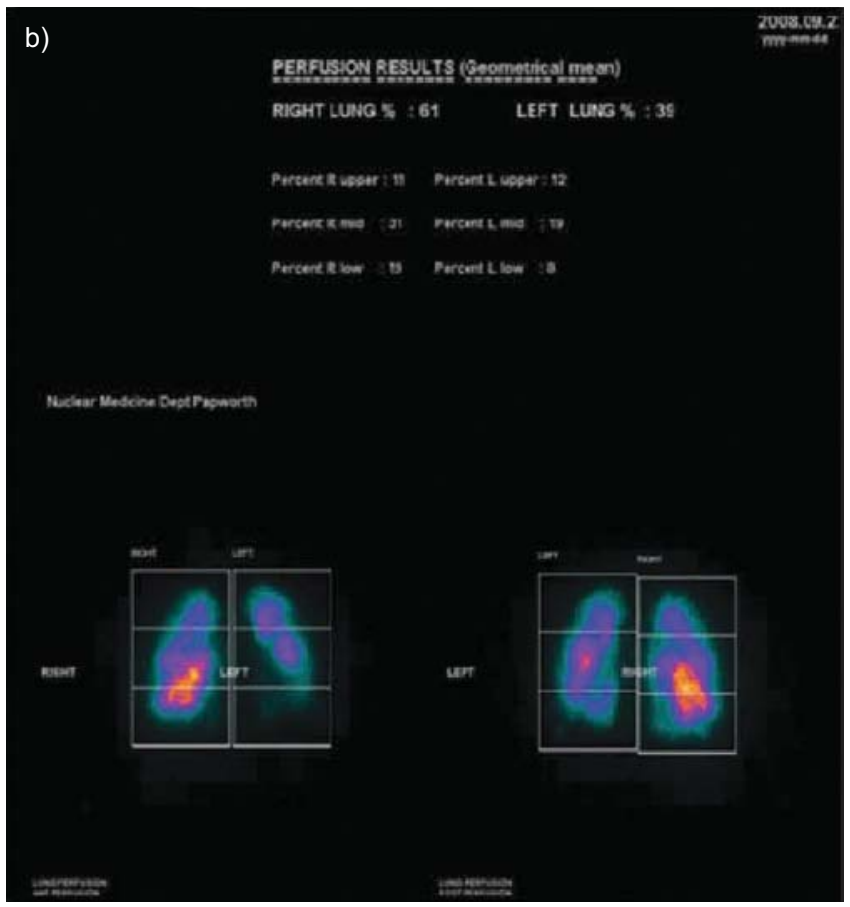

FIGURE 1. Pre-transplant a) chest radiograph and b) perfusion scan. 


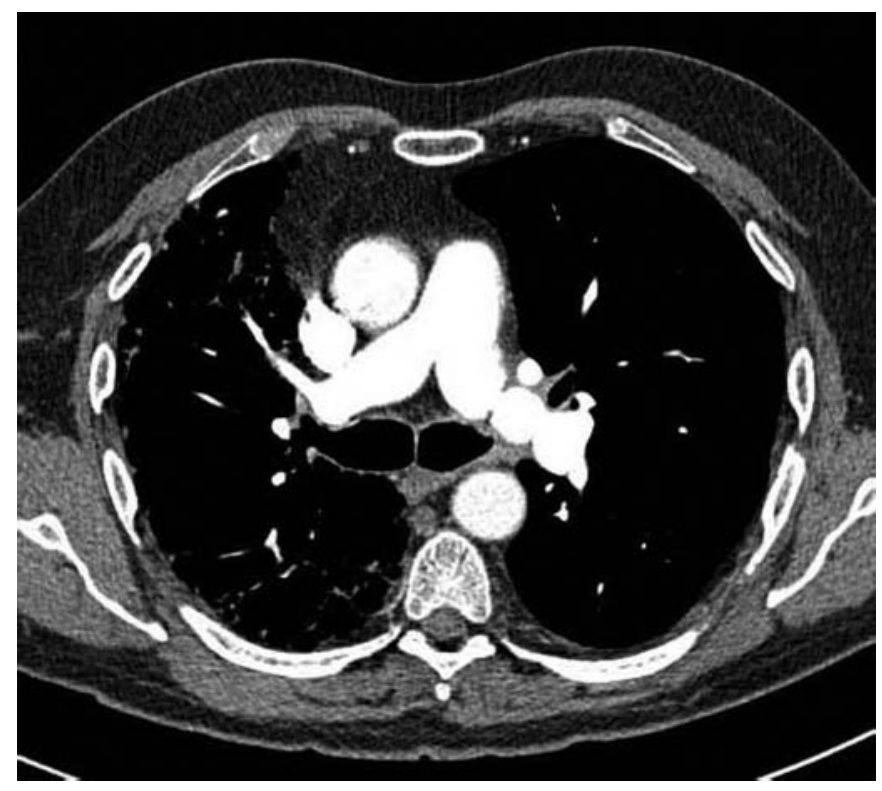

FIGURE 2. Computed tomography pulmonary angiogram showing pulmonary artery stricture.

surgical treatment of the stenosis is the preferred option but may still carry a poor prognosis. However, later the options are broader and include percutaneous intervention. We report a case of stenosis of the left main pulmonary artery following single lung transplantation, which was successfully treated by percutaneous transluminal balloon angioplasty and stent placement.

A 63-yr-old male with history of usual interstitial pneumonitis underwent a single left lung transplant in July 2009 (fig. 1). He also had a previous history of hepatitis B exposure. He had an

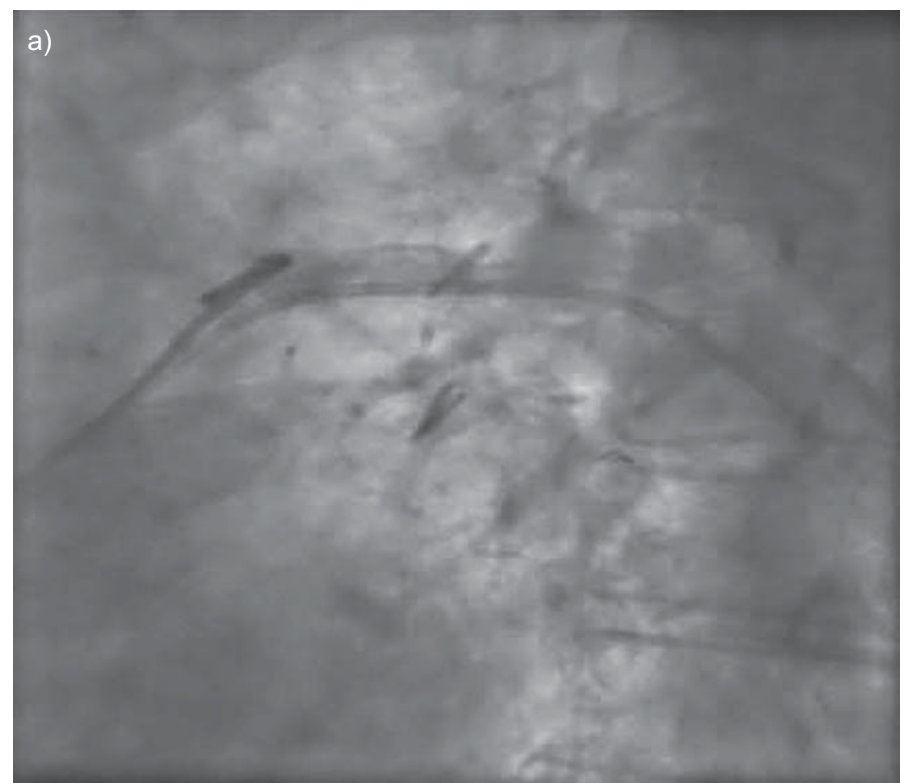

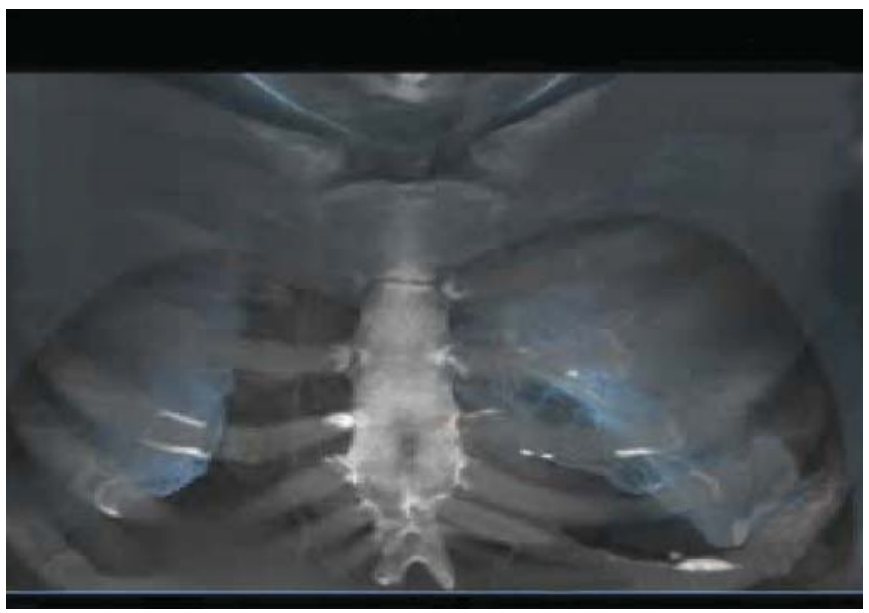

FIGURE 3. Post-transplant computed tomography pulmonary angiogram showing reduced perfusion to transplanted lung.

uncomplicated. post-operative course. Standard immunosuppresion was initiated with cyclosporine, mycophenolate and prednisolone. Fibre-optic bronchoscopy (FOB) performed at 3 weeks showed aspergillus and transbronchial biopsy revealed A1 rejection. He was treated with oral and inhaled anti-fungals and increased doses of oral prednisolone $(30 \mathrm{mg})$. He was discharged from hospital at 3 weeks post-transplant feeling well.

3 months post transplant, he presented with gradual onset of increasing breathlessness and worsening exercise tolerance. Spirometry revealed an FEV1 of $2.49 \mathrm{~L}$ and a forced vital capacity (FVC) of 3.29 L. A repeat FOB showed no evidence of infection or rejection. Plasma cytomegalovirus (CMV) PCR was elevated $\left(1.6 \times 10^{6} \mathrm{CMV}\right.$ copies $\left.\cdot \mathrm{mL}^{-1}\right)$ and treated with intravenous gancyclovir. 3 weeks of intravenous treatment reduced the CMV PCR to $<300$ copies $\cdot \mathrm{mL}^{-1}$ without any significant

FIGURE 4. Cheatham platinum stent placing a) pre and b) post angiogram.

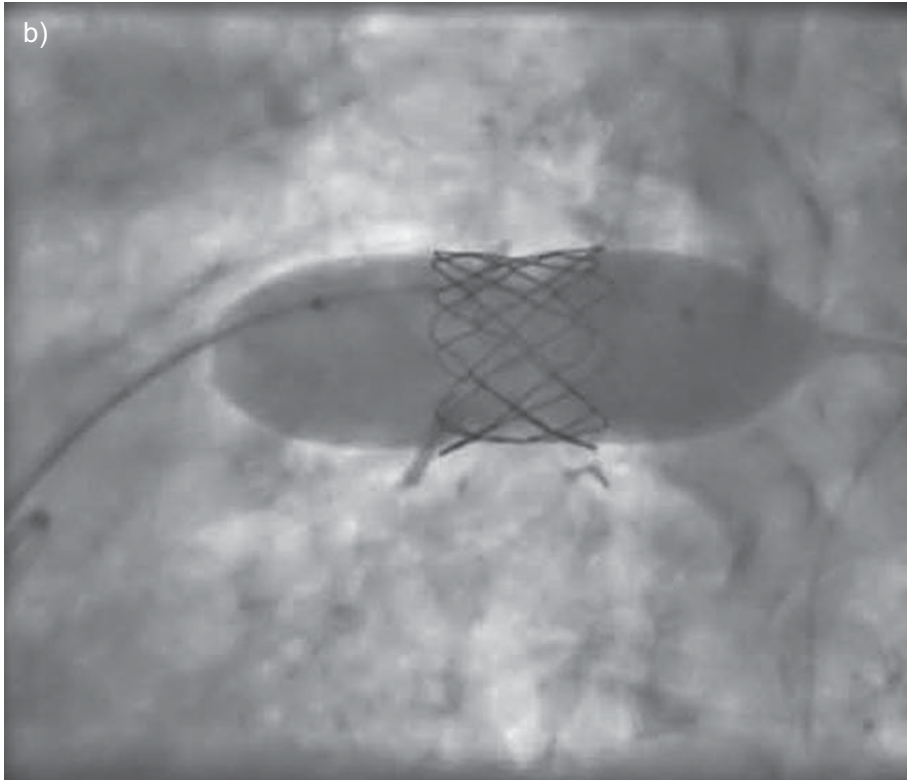




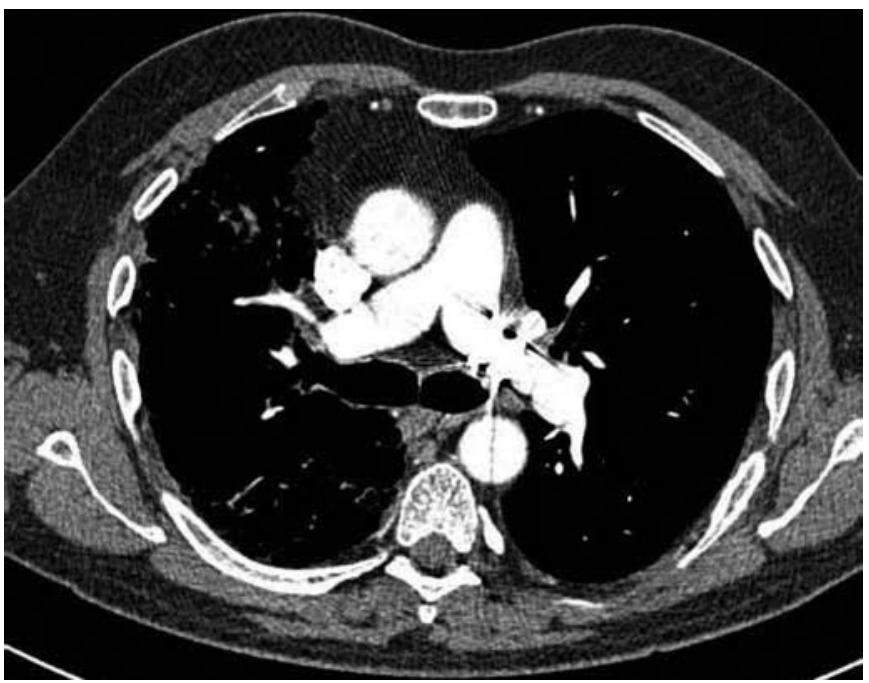

FIGURE 5. Post-stent correction of pulmonary artery stricture.

improvement in clinical symptoms. A CTPA revealed the presence of small pulmonary emboli (PE) in the left main pulmonary artery with infarction and minor change in the pulmonary artery calibre. A therapeutic INR was achieved and maintained through regular monitoring.

Despite these therapeutic measures the patient continued to complain of decreased exercise tolerance without spirometric change. 6 months post-transplant, the patient's breathlessness suddenly worsened and his exercise tolerance was limited to pre-transplant levels. Although his resting oxygen saturation was $95 \%$, post-exercise it dropped to $67 \%$. A further CTPA revealed no PE but severe stricturing of the pulmonary artery at the anastomtic site (fig. 2) with reduced perfusion to the transplanted lung (fig. 3).

A pulmonary arteriogram revealed a concentric narrowing in the left main pulmonary artery. The pressure gradient across the lesion was $10-12 \mathrm{mmHg}$. The pulmonary artery was initially balloon dilated and an $8 \times 20 \mathrm{~mm}$ Cheatham platinum stent was inserted (fig. 4). Post-stent placement the pressure gradient fell to $4 \mathrm{mmHg}$, with instant relief of symptoms.

2 weeks following stent insertion a repeat CTPA revealed resolution of the narrowing on the left main pulmonary artery (fig. 5). His 6-min walk distance improved to $500 \mathrm{~m}$, with little change in saturation from 96 to $91 \%$ during the test. His spirometry has remained unchanged with an FEV1 of $2.64 \mathrm{~L}$ and an FVC of $3.96 \mathrm{~L}$. The patient continues to remain well 18 months post-lung transplantation.

Single lung transplantation is an effective treatment for endstage pulmonary fibrosis. Dyspnoea and hypoxaemia in posttransplant patients are usually related to rejection or infection. Pulmonary artery anastomotic stenosis is a rare but serious complication defined as an anastomotic diameter of $<75 \%$ of that of the neighbouring vessels [3]. Other potential complications include kinking of the donor pulmonary artery distal to the anastomosis secondary to torsion [4]. The time of presentation varies from immediate post-operative period to a few years. The clinical features in the immediate post-operative period include refractory respiratory failure and persistent pulmonary hypertension. Delayed features include worsening dyspnoea and exercise tolerance [5]. Most cases present within the first 6 weeks post transplantation. In our case, the patient presented with symptoms early in the post-operative period. However, the $\mathrm{CMV}$ infection followed by the diagnosis of pulmonary embolism complicated the picture and delayed the diagnosis.

No single test is definitive and a variety of investigative diagnostic options are available. Trans-oesophageal echocardiogram provides important information regarding the size and patency of the proximal left pulmonary artery. Multi-detector CTPA with multi-planar reconstruction has improved the sensitivity and specificity of identifying vascular abnormalities in both the venous and arterial system. However, pulmonary angiogram remains the gold standard for examination of the pulmonary arterial tree [6].

Over time, the decrease in the native lung size and increase in the transplanted lung size can result in changes in the anatomical position of the anastomosis which may result in the anastomotic kinking or twisting producing a similar syndrome. This is unlikely to be the case in our patient as the stricture was at the anastomotic site and most probably represents a technical complication.

Although the long-term prognosis in our patient remains uncertain, the percutaneous insertion of a stent restored perfusion to the transplanted lung and avoided the risk of further surgery. However, vascular stents are associated with some complications including self limiting haemorrhage, stent migration, neo-intimal in growth and embolisation [6].

In contrast to the high risks posed by open surgery, endovascular techniques are simple and well tolerated and offer an additional treatment option for this rare complication $[7,8]$.

\section{S.K. Banerjee, K. Santhanakrishnan, L. Shapiro, J. Dunning,} S. Tsui and J. Parmar

Transplant Continuing Care Unit, Transplant Unit, Papworth Hospital NHS Trust, Papworth Everard, UK.

Correspondence: S. Banerjee, Transplant Continuing Care Unit, Transplant Unit, Papworth Hospital NHS Trust, Papworth Everard, Cambridgeshire, CB23 3RE, UK. E-mail: sandipbanerjee74@gmail.com

\section{Statement of Interest: None declared.}

Provenance: Submitted article, peer reviewed.

\section{REFERENCES}

1 Grossman RF, Frost A, Zamel N, et al. Results of single-lung transplantation for bilateral pulmonary fibrosis. The Toronto Lung Transplant Group. N Engl J Med 1990; 322: 727-733.

2 Christie JD, Edwards LB, Aurora P, et al. Registry of the International Society for Heart and Lung Transplantation: twentyfifth official adult lung and heart/lung transplantation report 2008. J Heart Lung Transplant 2008; 27: 957-969.

3 Ferreti G, Boutelant M, Thony F, et al. Successful stenting of a pulmonary arterial stenosis after a single lung transplant. Thorax 1995; 50: 1011-1012. 
4 Shoji T, Hanaoka N, Wada H, et al. Balloon angioplasty for pulmonary artery stenosis after lung transplantation. Eur J Cardiothorac Surg 2008; 34: 693-694.

5 Waurick PE, Kleber FX, Ewert R, et al. Pulmonary artery stenosis 5 years after single lung transplantation in primary pulmonary hypertension. J Heart Lung Transplant 1999; 18: 1243-1245.

6 Doyle T, Loyd J, Robbins IM. Percutaneous pulmonary artery and vein stenting. Am J Respir Crit Care Med 2001; 164: 657-660.
7 Gaubert J, Moulin G, Thomas P, et al. Anastomotic stenosis of the left pulmonary artery after lung transplantation: treatment by percutaneous placement of an endoprosthesis. AJR Am J Roentgenol 1993; 161: 947-949.

8 Berger H, Steiner W, Schmidt D, et al. Stent-angioplasty of an anastomotic stenosis of the pulmonary artery after lung transplantation. Eur J Cardiothorac Surg 1994; 8: 103-105.

DOI: $10.1183 / 09059180.00009610$

\section{Tobacco smoking: why do physicians not make diagnoses?}

\section{To the Editor:}

Tobacco smoking is recognised as the single greatest cause of avoidable morbidity and mortality in developed countries. An enormous amount of scientific evidence to support this statement has been collected in the past 60 yrs. Searching for "tobacco smoking and diseases" through PubMed provides results for 62,976 papers published since 1950. Notwithstanding this, physicians often forget that tobacco smoking, in addition to being a cause of many different diseases, is a disease itself.

In 1980, for the first time in the history of disease classification, the diagnosis of tobacco dependence was included in the third edition of the Diagnostic and Statistical Manual of Mental Disorders (DSM).

In 1988, the US Surgeon General issued a seminal report entitled "Nicotine Addiction", in which it was clearly stated that cigarettes were addictive, nicotine was the drug in tobacco that causes addiction, and tobacco addiction was similar to other substance addictions, such as to heroin or cocaine.

In 1992, the World Health Organization (WHO) included tobacco smoking under the "Mental and Behavioural Disorders" and, in the International Classification of Diseases 10, tobacco dependence has been defined as "a cluster of behavioural, cognitive and physiological phenomena that develop after repeated use".

In 1994, the DSM IV referred to nicotine dependence and nicotine withdrawal as psychiatric disorders, and the DSM IV Text Revised (DSM IV-TR) introduced the section of nicotine use disorders, including nicotine dependence, nicotine-induced disorders and nicotine-related disorders not otherwise specified.

In 2007, the European Respiratory Society (ERS) Task Force on smoking cessation in patients with respiratory diseases stated that tobacco smoking/nicotine addiction can be regarded as a chronic, recurrent disease; that dependence on tobacco is a complex behaviour, with both environmental and genetic influences; and that nicotine is the main component in cigarettes that contributes to addiction, although psychological factors and habituation also play a role [1].

Over time, tobacco smoking has been demonstrated to be a disease associated with younger age, lower income, reduced educational achievement and disadvantaged neighbourhood environment [2]. Moreover, risk factors increasing susceptibility to tobacco dependence have been well documented: starting smoking early in adolescence; comorbidity with mental illnesses or substance abuse disorders; and genetic-based rapid metabolism of nicotine [3].

If tobacco smoking has been definitively defined as a disease, why do doctors not usually make this diagnosis?

Different demographic or socio-economic conditions associated with the patient may reduce the probability that a smoker will be diagnosed. For example, patients of low socio-economic classes may have greater difficulty in accessing healthcare, even in some developed countries. However, most barriers in diagnosing tobacco dependence appear, surprisingly, to be related just to physicians. These can be summarised as follows.

1) Cultural heritage. Health personnel often use corroborated terms rather than the more new and appropriate ones. This cultural heritage seems to exist in different medical disciplines. However, for other pathological conditions, the use of an old term has not prevented the formulation the proper diagnosis. For instance, "absence seizure", introduced in 1981 by the Commission on Classification and Terminology of the International League against Epilepsy, is a diagnosis used also by those clinicians who still name the diseases as "petit mal seizures", a French term dating from the late 1700s.

2) Medical school training. In some countries (e.g. Italy, Spain, UK and Belgium), medical students are still trained to collect data on tobacco smoking (e.g. cigarettes smoked daily and packyears) in the physiological part of the case history. However, as a result of personal communications with other physicians, we note that in other countries (e.g. India and Croatia), tobacco dependence is correctly included among other concomitant diseases in the pathological part of the case history.

3) Reticence in recognising tobacco dependence as a relevant health problem. The health community seems to neglect tobacco dependence, not only when patients' health problems are considered, but also when their own health is considered. A high prevalence of smoking among physicians may explain this attitude. Although only 3\% of Canadian physicians [4], 4.4\% of ERS members [5], and $12 \%$ of Swiss primary care physicians currently smoke cigarettes [6], almost one-third (32.1\%) of French physicians [7] and $38.6 \%$ of Greek physicians are current smokers [8]. In Italy, the overall prevalence of smoking among hospital staff is $30.6 \%$ and among physicians is $21.2 \%$; moreover, only $58.7 \%$ of hospital health staff tackles the issue of smoking in 